\title{
LOVE WAVES IN STRATIFIED MONOCLINIC MEDIA
}

\author{
BY \\ SERGEY V. KUZNETSOV \\ Institute for Problems in Mechanics, Prosp. Vernadskogo, 101, Moscow, 119526 Russia
}

\begin{abstract}
A mathematical model for analysis of Love waves propagating in stratified anisotropic (monoclinic) media is presented; this model is based on a newly developed Modified Transfer Matrix (MTM) method. Closed form dispersed relations are obtained for media consisting of one or two orthotropic layers lying on orthotropic substrate. Conditions for existence of Love waves are analyzed. Horizontally polarized shear surface waves of non-Love type are constructed. A numerical algorithm is worked out for obtaining dispersion relations for Love waves propagating in stratified media containing a large number of layers.
\end{abstract}

1. Introduction. Love [1] has shown that under certain conditions surface waves with horizontal transverse polarization can propagate in a system composed of a layer contacting with a substrate, of which both were assumed to be elastic and isotropic. As is typical for other surface waves, it was supposed that in the substrate Love wave attenuated with depth. Along with Rayleigh waves, Love waves play an important part in transmitting seismic energy in earthquakes, and quite often these waves are recoded at the seismic activity and explosions.

The displacement ficld corresponding to Love wave can be represented in the form

$$
\left\{\begin{array}{l}
\mathbf{u}_{1}(\mathbf{x})=\mathbf{m}\left(C_{1} e^{-i r \gamma_{1} x^{\prime}}+C_{2} e^{i r \gamma_{1} x^{\prime}}\right) e^{i r(\mathbf{n} \cdot \mathbf{x}-c t)} \\
\mathbf{u}_{2}(\mathbf{x})=\mathbf{m}\left(C_{3} e^{i r \gamma_{2} x^{\prime}}\right) e^{i r(\mathbf{n} \cdot \mathbf{x}-c t)}
\end{array}\right.
$$

where $\mathbf{u}_{1}$ and $\mathbf{u}_{2}$ refer to the displacements in the layer and substrate respectively; $\mathbf{m}$ is the unit amplitude (polarization) vector, assumed to be normal to both the direction of propagation $\mathbf{n}$ and unit normal $\nu$ to the interface surface; $x^{\prime} \equiv \nu \cdot \mathbf{x}$ is the coordinate along a direction determined by the vector $\nu$ (it will be assumed further that $x^{\prime}$ is negative in the substrate); $r$ is the wave number; $c$ is the phase speed; $t$ is time; and the unknown (complex) coefficients $C_{k}$ are determined up to a multiplier by the traction-free boundary conditions at the outer plane boundary:

$$
\left.\mathbf{t}_{\nu}\right|_{x^{\prime}=h} \equiv \nu \cdot \mathbf{C}_{1} \cdots \nabla_{\mathbf{x}} \mathbf{u}_{1}=0
$$

Received October 26, 2003.

2000 Mathematics Subject Classification. Primary 74J15.

Key words and phrases. Love wave, surface wave, anisotropy, laminated medium. 
and the contact-type conditions at the interface $\left(. x^{\prime}=0\right)$ :

$$
\left\{\begin{array}{l}
\nu \cdot \mathbf{C}_{1} \cdots \nabla_{\mathbf{x}} \mathbf{u}_{1}=\nu \cdot \mathbf{C}_{2} \cdots \nabla_{\mathbf{x}} \mathbf{u}_{2} \\
\mathbf{u}_{1}=\mathbf{u}_{2} .
\end{array}\right.
$$

Parameters $i k, k=1,2$ in (1.1) correspond to complex roots of the Christoffel equation; this equation will be introduced later. In Eqs. (1.2), (1.3), $\mathbf{C}_{k}, k=1,2$ are the fourth-order elasticity tensors related to the layer and substrate respectively and $h$ is the thickness of the layer.

REMARK 1.1. According to representation (1.1) attenuation with depth in the substrate is ensured by the Christoffel parameter $\gamma_{2}$ with negative imaginary part; this will be assumed.

The following proposition takes place:

\section{Proposition 1.1.}

1) Love waves can arise in an isotropic laver and the contacting substrate if and only if the phase speed satisfics the following relation:

$$
c_{1}^{T}<c<c_{2}^{T} .
$$

where $c_{k}^{T}=\sqrt{\frac{\mu_{k}}{\rho_{k}}}, k=1,2$ volocities of the transverse bulk waves in a layer and substrate respectively, and $\mu_{k}$ and $\rho_{k}$ are the corresponding Lame's constants and densities.

2) Dispersion relation between the phase speed $c$ and frequency $w$ admits the following representation:

$$
\omega=\frac{c}{h}\left(\frac{\rho_{1} c^{2}}{\mu_{1}}-1\right)^{-1 / 2}\left(\arctan \left(\frac{\mu_{2}}{\mu_{1}}\left(\frac{1-\frac{\rho_{2} c^{2}}{\mu_{2}}}{\frac{\rho_{1} c^{2}}{\mu_{1}}-1}\right)^{1 / 2}\right)+n \pi\right), n=0,1,2, \ldots
$$

Corollary 1.

a) At the fixed frequency $w$ there is a finite number of Love waves propagating with different phase speeds $c \in\left(c_{1}^{T} ; c_{2}^{T}\right)$.

b) At the fixed phase speed $c \in\left(c_{1}^{T} ; c_{2}^{T}\right)$ there is denumerable number of Love waves propagating with different frequencies $\omega$.

Corollary 2. No Love waves can propagate if $c_{1}^{T}>c_{2}^{T}$.

Dieulesaint and Royer [2] have shown that Love waves can also propagate in a system composed of anisotropic layer lying on a substrate, both of which possess axes of elastic symmetry of the fourth- or sixth-order in the transverse direction coinciding with the amplitude vector $\mathbf{m}$. For such a system, condition of propagation and the dispersion relation resemble relations (1.4) and (1.5). Some modifications of the Dieulesaint and Royer approach for surface waves in the transversely isotropic layer and substrate can be found in a recent paper [3].

For stratified medium containing more than one layer on a substrate, there are no analytical solutions similar to (1.5). Love waves in such a medium can be studied numerically by applying two different matrix approaches originally developed for analysis of Lamb waves propagating in isotropic layers and substrates. These approaches are 
known as the Transfer Matrix (TM) method (sometimes this method is referred to as the Thomson-Haskell method due to their originators [4, 5]), and the Global Matrix (GM) method proposed in $[6,7]$. The TM-method is based on the successive soluiions of the contact-type boundary conditions at the interfaces and constructing the corresponding transfer matrices; this method will be discussed in detail later. The origin of the GM-method is based on the solution of ordinary differential equations with piecewise continuous coefficients for the layered structure, resulting in construction of the spccial "global" matrix.

Since it appeared, the GM-method proved to be more numerically stable than its rival, especially for the case of high frequencies and large thickness of some layers. This situation is known as the "large $f d$-problem", where $f$ is frequency and $d$ is thickness of a layer. Some improvements of the TM-method towards its better numerical stability were suggested also. Mainly, these improvements are based on rearranging the secular equations and corresponding matrices in such a way that they do not form an ill-posed problem. Dunkin [8] delivers one of the first approaches in that direction; see also [9]-[11]. However, the TM-method being applied to analysis of Love waves in media with relatively small number of layers yields to the GM-method in numerical stability, but performs the latter in computation speed; see $[12,13]$ for discussions. The problem becomes more complicated when we arrive at a medium composed of a large number of layers. Because of the necessity to analyze degeneracy of the determinants of the rank $2 n+1$ where $n$ is the number of layers, the GM-method becomes useless for studying Love waves in media with relatively large number of layers (due to numerical errors the determinants become highly oscillating in the vicinity of zeroes). To improve this situation, decomposition of the global matrix and analysis of the smallest eigenvalues of the global matrix rather than analyzing the determinant was proposed in [14].

In the present paper, the Modified Transfer Matrix (MTM) method is being developed for analysis of Love waves propagating in stratified anisotropic media with monoclinic symmetry. This method appears to be rather fast, as is typical for all realizations of the TM-method, and at the same time it possesses good numerical stability due to (i) choosing floating origins for the displacement fields in layers, and (ii) using a modified representation for Love waves in layers based on hyperbolic functions. As will be demonstrated, the MTM method can equally be applied to obtaining analytical solutions for media containing one or two layers, and to numerical analysis of Love waves propagating in stratified media containing a large number of layers.

It will also be shown that representation (1.1) is not valid for the case of multiple roots of the Christoffel equation, and the correct representation for such a case will be constructed, yielding surface waves of non-Love type. The MTM method will also be used for obtaining secular equations for surface waves with horizontal shear polarization and propagating in laminated plates with the traction-free, clamped, and mixed boundary conditions. 
2. Basic notations. In the subsequent analysis both layers and the substrate are assumed to be homogeneous and lincarly hyperelastic. Equations of motion for homogeneous anisotropic clastic medium can be written in the form

$$
\mathbf{A}\left(\partial_{x} . \dot{\partial}_{t}\right) \mathbf{u} \equiv \operatorname{div}_{x} \mathbf{C} \cdots \nabla_{x} \mathbf{u}-\rho \ddot{\mathbf{u}}=0
$$

where elasticity tensor $\mathbf{C}$ is assumed to be positive definite:

$$
\underset{A \in \sin \left(R^{3} \subseteq R^{3}\right), \quad \mathbf{A} \neq 0}{\forall \mathbf{A}}(\mathbf{A} \cdot \mathbf{C} \cdot \mathbf{A}) \equiv \sum_{i, j \cdot m . n} A_{i j} C^{i j m n} A_{m n}>0 .
$$

REMARK 2.1. a) The other assumption concerms symmetry of the elasticity tensor. It will be assumed further that all the regarded materials possess planes of elastic symmetry coinciding with the sagittal plane $\mathbf{m} \cdot \mathbf{x}=0$. This assumption is provided by the elasticity tensor belonging to the monoclinic system. and it is equivalent to vanishing all of the decomposable components of the tensor $\mathbf{C}$ having odd number of entries of vector $\mathbf{m}$ (in the orthogonal basis in $R^{3}$ generated by vector $\mathbf{m}$ and any two orthogonal vectors belonging to the sagittal plane). For monoclinic symmetry, the elasticity tensor in the regarded basis has 13 independent decomposable components; see [15].

b) It will be shown later that assuming monoclinic symmetry provided a sufficient condition for the surface tractions acting on any plane $\nu \cdot \mathbf{x}=$ const to be collinear with vector $\mathbf{m}$.

Following [16, 17], a more general representation for Love wave than (1.1) will be considered:

$$
\mathbf{m} f\left(i r x^{\prime}\right) e^{i r(\mathbf{n} \cdot \mathbf{x}-c t)} .
$$

where $x^{\prime}=\nu \cdot \mathbf{x}$ as in (1.1): $f$ is the unknown scalar function: the exponential multiplier in (2.3) corresponds to propagation of plane wave front along direction $\mathbf{n}$ with the phase speed $c$; and $r$ is the wave number. Substituting representation (2.3) into Eq. (2.1) and taking into account Remark 2.1.a. yields the following differential equation:

$$
\begin{aligned}
& \left(\begin{array}{r}
(\mathbf{m} \otimes \nu \cdots \mathbf{C} \cdot \nu \otimes \mathbf{m}) \partial_{x^{\prime}}^{2}+(\mathbf{m} \otimes \nu \cdot \mathbf{C} \cdot \mathbf{n} \otimes \mathbf{m}+\mathbf{m} \otimes \mathbf{n} \cdots \mathbf{C} \cdot \nu \otimes \mathbf{m}) \partial_{x^{\prime}}+ \\
\left(\mathbf{m} \otimes \mathbf{n} \cdots \mathbf{C} \cdot \mathbf{n} \otimes \mathbf{m}-\rho c^{2}\right)
\end{array}\right) f\left(i r \cdot x^{\prime}\right) \\
& =0 \text {. }
\end{aligned}
$$

The characteristic equation for the differential equation (2.4), known also as the Christoffel equation, has the form:

$$
\begin{array}{r}
(\mathbf{m} \otimes \nu \cdot \mathbf{C} \cdot \nu \otimes \mathbf{m}) \gamma^{2}+(\mathbf{m} \otimes \nu \cdot \mathbf{C} \cdot \mathbf{n} \otimes \mathbf{m}+\mathbf{m} \otimes \mathbf{n} \cdot \mathbf{C} \cdot \nu \otimes \mathbf{m}) \gamma+\left(\mathbf{m} \otimes \mathbf{n} \cdot \mathbf{C} \cdot \mathbf{n} \otimes \mathbf{m}-\rho c^{2}\right) \\
=0 . \quad(2.5)
\end{array}
$$

The left-hand side of Eq. (2.5) represents a polynomial of degree 2 with respect to the Christoffel parameter $\gamma$. and thus. for the regarded elastic symmetry only two partial waves composing Love wave in a layer can be present.

3. Displacements and surface tractions in the substrate. In the following analysis it is assumed that the medium consists of $n$ layers lying on a substrate: the lower 
index $n+1$ will be referred to as the substrate. Since the displacement field in the substrate must attenuate with depth, the appropriate root of Eq. (2.5) should be complex and with negative imaginary part; see Remark 1.1.

The following proposition takes place:

Proposition 3.1. Attenuation with depth in the monoclinic substrate is possible, if and only if the phase speed $c$ belongs to the (non-empty) speed interval:

$$
c \in\left(0 ; \sqrt{\rho_{n+1}^{-1}\left(\mathbf{m} \otimes \mathbf{n} \cdot \mathbf{C}_{n+1} \cdot \mathbf{n} \otimes \mathbf{m}-\frac{\left(\mathbf{m} \cdot \operatorname{sym}\left(\mathbf{n} \cdot \mathbf{C}_{n+1} \cdot \nu\right) \cdot \mathbf{m}\right)^{2}}{\left(\mathbf{m} \otimes \nu \cdot \mathbf{C}_{n+1} \cdot \nu \otimes \mathbf{m}\right)}\right)}\right),
$$

where for any second-order tensor $\mathbf{A}: \operatorname{sym}(\mathbf{A}) \equiv \frac{1}{2}\left(\mathbf{A}+\mathbf{A}^{t}\right)$. For the speed interval (3.1) the corresponding Christoffel parameter $\gamma_{n+1}$ is complex with negative imaginary part:

$$
\begin{aligned}
\gamma_{n+1}=- & \frac{\mathbf{m} \cdot \operatorname{sym}\left(\mathbf{n} \cdot \mathbf{C}_{n+1} \cdot \nu\right) \cdot \mathbf{m}}{\mathbf{m} \otimes \nu \cdot \mathbf{C}_{n+1} \cdot \nu \otimes \mathbf{m}} \\
& -i \sqrt{\frac{\mathbf{m} \otimes \mathbf{n} \cdot \mathbf{C}_{n+1} \cdot \mathbf{n} \otimes \mathbf{m}-\rho_{n+1} c^{2}}{\mathbf{m} \otimes \nu \cdot \mathbf{C}_{n+1} \cdot \nu \otimes \mathbf{m}}-\left(\frac{\mathbf{m} \cdot \operatorname{sym}\left(\mathbf{n} \cdot \mathbf{C}_{n+1} \cdot \nu\right) \cdot \mathbf{m}}{\mathbf{m} \otimes \nu \cdot \mathbf{C}_{n+1} \cdot \nu \otimes \mathbf{m}}\right)^{2}} .
\end{aligned}
$$

Proof. Direct analysis of roots of Eq. (2.5) reveals that these roots are complex, provided the corresponding discriminant is negative, and this gives the upper bound in (3.1). It remains to show that the radicand in (3.1) is positive. But, this flows out from the analysis of the quadratic polynomial:

$$
P(x) \equiv \mathbf{m} \otimes(x \nu+\mathbf{n}) \cdots \mathbf{C}_{n+1} \cdots(\mathbf{n}+x \nu) \otimes \mathbf{m} .
$$

The right-hand side in (3.3) is positive at any real $x$, due to the assumed positive definiteness of the elasticity tensor. Condition of non-existence of real roots of this polynomial completes the proof, since the discriminant of this polynomial coincides (up to a multiplier $\left.-\left(\rho_{n+1}\right)^{-1 / 2}\right)$ with the upper bound in (3.1). Expression (3.2) is obtained by solving Eq. (2.5).

Corollary 1. Parameter $\gamma_{n+1}$ cannot be multiple root of the Christoffel equation (2.5).

Proof flows out from non-vanishing discriminant in (3.2), which is necessary for attenuation of the surface wave with depth.

COROLlary 2. If the regarded material possesses another plane of elastic symmetry with the unit normal coinciding with vector $\mathbf{n}$ or $\nu$ (such a material is necessarily orthotropic), then the admissible speed interval becomes:

$$
c \in\left(0 ; c_{n+1}^{T}\right),
$$

where $c_{n+1}^{T}$ is the speed of the transverse bulk wave in substrate propagating in direction $\mathbf{n}$ and having amplitude vector $\mathbf{m}$. For the regarded case the corresponding Christoffel parameter $\gamma_{n+1}$ is purely imaginary:

$$
\gamma_{n+1}=-i \sqrt{\frac{\mathbf{m} \otimes \mathbf{n} \cdot \mathbf{C}_{n+1} \cdots \mathbf{n} \otimes \mathbf{m}-\rho_{n+1} c^{2}}{\mathbf{m} \otimes \nu \cdot \mathbf{C}_{n+1} \cdots \nu \otimes \mathbf{m}}} .
$$


Proof. For such a material the term $\left(\operatorname{sym}\left(\mathbf{m} \otimes \mathbf{n} \cdots \mathbf{C}_{n+1} \cdots \nu \otimes \mathbf{m}\right)\right)$ in $(3.2)$ vanishes, since it contains odd entries of vectors $\mathbf{n}$ and $\nu$. Now, it is sufficient to note that the remaining radicand in $(3.1)$ coincides with the speed $c_{n+1}^{T}$. The rest of this proof flows out from Eq. (2.5).

Representation (1.1) for the substrate leads to the following field of surface tractions acting on the plane $\nu \cdot \mathbf{x}=0$ :

$$
\left.\mathbf{t}_{n+1}(\mathbf{x})\right|_{\nu \cdot \mathbf{x}=0}=i r C_{2 n+1}\left(\gamma_{n+1}\left(\nu \cdot \mathbf{C}_{n+1} \cdot \nu \otimes \mathbf{m}\right)+\left(\nu \cdot \mathbf{C}_{n+1} \cdot \mathbf{n} \otimes \mathbf{m}\right)\right) e^{i r(\mathbf{n} \cdot \mathbf{x}-c t)} .
$$

PROPOSITION 3.2. Surface traction field (3.6) is collinear with vector $\mathbf{m}$.

Proof. Flows out from the assumed monoclinic symmetry with respect to vector $\mathbf{m}$, which ensures even number of entering vector $\mathbf{m}$ in the decomposable components of the tensor $\mathbf{C}_{n+1}$ in the basis generated by vectors $\mathbf{m}, \nu, \mathbf{n}$. Thus, both vectors $\left(\nu \cdot \mathbf{C}_{n+1} \cdot \nu \otimes \mathbf{m}\right)$ and $\left(\nu \cdot \mathbf{C}_{n+1} \cdot \mathbf{n} \otimes \mathbf{m}\right)$ in the right-hand side of (3.6) are collinear with vector $\mathbf{m}$.

4. Displacements and surface tractions in layers. In this section the lower index $k(1 \leq k \leq n)$ is referred to as the corresponding layer.

4.1. Aliquant roots. For aliquant roots of the Christoffel equation and orthotropic material with the principle elasticities coinciding with vectors $\mathbf{m}, \mathbf{n}$ and $\nu$, representation (1.1) remains valid. Still, for the current analysis, which includes a more general case of monoclinic materials, it is desirable to modify representation (1.1) in the following way:

$$
\mathbf{u}_{k}(\mathbf{x})=\mathbf{m}\left(C_{2 k-1} \sinh \left(i r \alpha_{k} x^{\prime}\right)+C_{2 k} \cosh \left(i r \alpha_{k} x^{\prime}\right)\right) e^{i r\left(\beta_{k} \cdot x^{\prime}+\mathbf{n} \cdot \mathbf{x}-c t\right)},
$$

where $\gamma_{k}=\alpha_{k}+\beta_{k}$, and

$$
\begin{aligned}
\alpha_{k} & =-i \sqrt{\frac{\mathbf{m} \otimes \mathbf{n} \cdot \mathbf{C}_{k} \cdot \mathbf{n} \otimes \mathbf{m}-\rho_{k} c^{2}}{\mathbf{m} \otimes \nu \cdot \mathbf{C}_{k} \cdot \nu \otimes \mathbf{m}}-\left(\frac{\mathbf{m} \cdot \operatorname{sym}\left(\mathbf{n} \cdot \mathbf{C}_{k} \cdot \nu\right) \cdot \mathbf{m}}{\mathbf{m} \otimes \nu \cdot \mathbf{C}_{k} \cdot \nu \otimes \mathbf{m}}\right)^{2}}, \\
\beta_{k} & =-\frac{\mathbf{m} \cdot \operatorname{sym}\left(\mathbf{n} \cdot \mathbf{C}_{k} \cdot \nu\right) \cdot \mathbf{m}}{\mathbf{m} \otimes \nu \cdots \mathbf{C}_{k} \cdot \nu \otimes \mathbf{m}} .
\end{aligned}
$$

Thus, $\alpha_{k}$ is real or imaginary depending on the value of the phase speed, and $\beta_{k}$ is real and independent of $c$.

Taking into account (4.1), the corresponding surface tractions acting on the plane $\nu \cdot \mathbf{x}=x^{\prime}$ are:

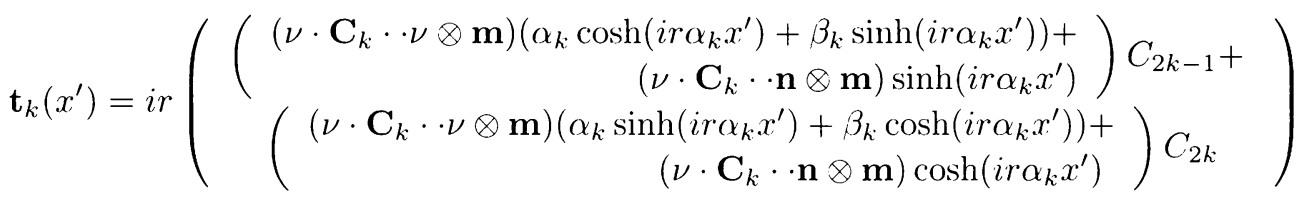

$$
\begin{aligned}
& \times e^{i r\left(\beta_{k} x^{\prime}+\mathbf{n} \cdot \mathbf{x}-c t\right)} .
\end{aligned}
$$

Proposition 4.1. Surface tractions (4.3) are collinear with vector $\mathbf{m}$.

Proof is analogous to the proof of Proposition 3.2.

Taking into account (4.3) and the fact that $\nu \cdot \mathbf{C}_{k} \cdots \mathbf{n} \otimes \mathbf{m}=0, \beta_{k}=0$, and $\gamma_{k}=\alpha_{k}$ for the orthotropic material with axes of elastic symmetry coinciding with vectors $\mathbf{m}$. $\mathbf{n}$, and 
$\nu$, we arrive at the following expression for the surface tractions acting in the orthotropic layer:

$$
\mathbf{t}_{k}\left(x^{\prime}\right)=i r \gamma_{k}\left(\nu \cdot \mathbf{C}_{k} \cdot \nu \otimes \mathbf{m}\right)\left(C_{2 k-1} \cosh \left(i r \gamma_{k} x^{\prime}\right)+C_{2 k} \sinh \left(i r \gamma_{k} x^{\prime}\right)\right) e^{i r(\mathbf{n} \cdot \mathbf{x}-c t)} .
$$

4.2. Multiple roots. Representation (4.1) for Love waves in a layer becomes incorrect when multiple roots of the Christoffel equation arise; see $[16,17]$ where appearing multiple roots are analyzed in detail for the case of Rayleigh waves.

Multiple roots arise when parameter $\alpha_{k}$ in (4.2) vanishes. This allows us to formulate the following proposition:

Proposition 4.2. a) The phase speed at which multiple roots arise is:

$$
c=\sqrt{\rho^{-1}\left(\mathbf{m} \otimes \mathbf{n} \cdot \mathbf{C}_{k} \cdot \mathbf{n} \otimes \mathbf{m}-\frac{\left(\mathbf{m} \cdot \operatorname{sym}\left(\mathbf{n} \cdot \mathbf{C}_{k} \cdot \nu\right) \cdot \mathbf{m}\right)^{2}}{\mathbf{m} \otimes \nu \cdot \mathbf{C}_{k} \cdot \nu \otimes \mathbf{m}}\right)} .
$$

b) The corresponding Christoffel parameter $\gamma_{k}$ (necessarily real) is:

$$
\gamma_{k}=-\frac{\mathbf{m} \cdot \operatorname{sym}\left(\mathbf{n} \cdot \mathbf{C}_{k} \cdot \nu\right) \cdot \mathbf{m}}{\mathbf{m} \otimes \nu \cdot \mathbf{C}_{k} \cdot \nu \otimes \mathbf{m}} .
$$

c) Representation for the displacement field corresponding to multiple roots is:

$$
\mathbf{u}_{k}(\mathbf{x})=\mathbf{m}\left(C_{2 k-1}+i r x^{\prime} C_{2 k}\right) e^{i r\left(\gamma_{k} x^{\prime}+\mathbf{n} \cdot \mathbf{x}-c t\right)} .
$$

d) The corresponding surface tractions on the plane $\nu \cdot \mathbf{x}=x^{\prime}$ are:

$$
\mathbf{t}_{k}\left(x^{\prime}\right)=i r\left(\begin{array}{r}
\left(\nu \cdot \mathbf{C}_{k} \cdot \nu \otimes \mathbf{m}\right)\left(\gamma_{k} C_{2 k-1}+\left(1+i r \gamma_{k} x^{\prime}\right) C_{2 k}\right)+ \\
\left(\nu \cdot \mathbf{C}_{k} \cdot \mathbf{n} \otimes \mathbf{m}\right)\left(C_{2 k-1}+i r x^{\prime} C_{2 k}\right)
\end{array}\right) e^{i r\left(\gamma_{k} x^{\prime}+\mathbf{n} \cdot \mathbf{x}-c t\right)} .
$$

Proof. Conditions a) and b) flow out from considering vanishing discriminant in (3.2). Condition c) corresponds to the general solution of Eq. (2.4) at multiple roots; see [16][18].

Proposition 4.3. Surface tractions (4.8) are collinear with vector $\mathbf{m}$.

Proof is analogous to the proof of Proposition 3.2.

REMARK 4.1. Surface wave (4.7) corresponding to appearing multiple roots will be referred to as non-Love wave.

\section{Modified Transfer Matrix Method.}

5.1. Transfer matrices. According to Propositions 4.1 and 4.2, scalar amplitudes of the displacements and surface tractions acting in the $k$-th layer on the plane $\nu \cdot \mathbf{x}=x^{\prime}$ can be represented in the form

$$
\left(\begin{array}{c}
u_{k}\left(x^{\prime}\right) \\
t_{k}\left(x^{\prime}\right)
\end{array}\right)=\mathbf{M}_{k}\left(x^{\prime}\right) \cdot\left(\begin{array}{c}
C_{2 k-1} \\
C_{2 k}
\end{array}\right)
$$

where $u_{k}\left(x^{\prime}\right) \equiv\left|\mathbf{u}_{k}\left(x^{\prime}\right) e^{-i r(\mathbf{n} \cdot \mathbf{x}-c t)}\right|, t_{k}\left(x^{\prime}\right) \equiv\left|\mathbf{t}_{k}\left(x^{\prime}\right) e^{-i r(\mathbf{n} \cdot \mathbf{x}-c t)}\right|$ are the corresponding scalar amplitudes, and $\mathbf{M}_{k}$ is a $2 \times 2$ matrix. Taking into account expressions (4.1), (4.3), (4.5), and (4.6), matrix $\mathbf{M}_{k}$ takes the form: 
a) Aliquant roots

$$
\begin{aligned}
& \mathbf{M}_{k}\left(x^{\prime}\right)
\end{aligned}
$$

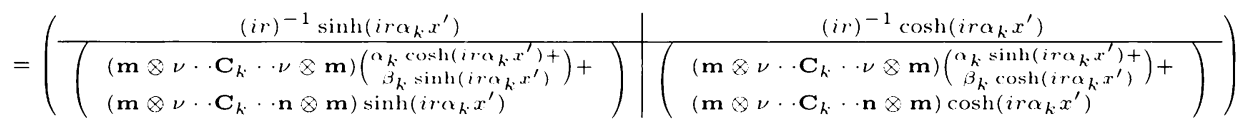

$$
\begin{aligned}
& \times \text { ire } e^{i n \cdot 3 k \cdot x^{\prime}}
\end{aligned}
$$

and

b) Multiple roots

$$
\mathbf{M}_{k}\left(x^{\prime}\right)=\left(\begin{array}{c|c}
(i r)^{-1} & x^{\prime} \\
\hline\left(\begin{array}{c}
\gamma_{k}\left(\mathbf{m} \otimes \mathbf{v} \cdot \mathbf{C}_{k} \cdot \nu \otimes \mathbf{m}\right)+ \\
\left(\mathbf{m} \otimes \nu \cdot \mathbf{C}_{k} \cdot \mathbf{n} \otimes \mathbf{m}\right)
\end{array}\right) & \left(\begin{array}{c}
\left(1+i r \gamma_{k} x^{\prime}\right)\left(\mathbf{m} \otimes \nu \cdot \mathbf{C}_{k} \cdot \nu \otimes \mathbf{m}\right)+ \\
i r x^{\prime}\left(\mathbf{m} \otimes \nu \cdot \cdot \mathbf{C}_{k} \cdot \mathbf{n} \otimes \mathbf{m}\right)
\end{array}\right)
\end{array}\right) i r e^{i r \gamma_{k} \cdot x^{\prime}} .
$$

Note that according to $(4.2) \beta_{k}$ in $(5.2)$ is independent of the phase speed $c$. The following proposition takes place:

Proposition 5.1. For both aliquant and multiple roots, matrices $\mathbf{M}_{k}$ are nonsingular at any real $x^{\prime}$.

Proof. At first we observe that the exponential multipliers $e^{i r \beta_{k} x^{\prime}}$ in (5.2) and $e^{i r \gamma_{k} x^{\prime}}$ in (5.3) are not zero at any $x^{\prime}$; then direct verification shows that matrices in the righthand sides of (5.2), (5.3) are not degenerate at any $x^{\prime}$ (the determinant for the matrix $(5.2)$ is $-\alpha_{k}\left(\mathbf{m} \otimes \nu \cdots \mathbf{C}_{k} \cdots \nu \otimes \mathbf{m}\right) e^{i r \beta_{k} x^{\prime}}$ with $\alpha_{k} \neq 0$, since the roots are aliquant; for the matrix (5.3) the corresponding determinant is $\left.\left(\mathbf{m} \otimes \nu \cdot \mathbf{C}_{k} \cdot \nu \otimes \mathbf{m}\right) e^{i \gamma_{k} \cdot x^{\prime}}\right)$. This completes the proof.

Now, by the use of transfer matrices $\mathbf{M}_{k}$, the displacements and surface tractions on the interface between the $n$-th layer and the substrate can be represented in terms of the unknown coefficients $C_{1}$ and $C_{2}$ only:

$$
\left(\begin{array}{l}
u_{n}\left(-h_{n} / 2\right) \\
t_{n}\left(-h_{n} / 2\right)
\end{array}\right)=\left(\prod_{k=2}^{n}\left(\mathbf{M}_{k}\left(-h_{k} / 2\right) \cdot \mathbf{M}_{k}^{-1}\left(h_{k} / 2\right)\right)\right) \cdot \mathbf{M}_{1}\left(-h_{1} / 2\right) \cdot\left(\begin{array}{l}
C_{1} \\
C_{2}
\end{array}\right),
$$

where $h_{k}, k=1, \ldots n$ is the thickness of the corresponding layer.

5.2. Boundary conditions at the outer boundary. Expressions (4.3), (4.8) allow us to formulate condition for the traction-free outer surface of the first layer in the following manner:

$$
t_{1}\left(h_{1} / 2\right) \equiv \vec{B}_{1}\left(h_{1} / 2\right) \cdot \vec{C}=0
$$

where $t_{1}$ is the scalar amplitude of the surface tractions; $\vec{B}_{1}\left(h_{1} / 2\right)=\left(X_{1}\left(h_{1} / 2\right) ; Y_{1}\left(h_{1} / 2\right)\right)$; and $\vec{C}=\left(C_{1} ; C_{2}\right)$. Components $X_{1}\left(h_{1} / 2\right)$ and $Y_{1}\left(h_{1} / 2\right)$ take the form: 
a) Aliquant roots

$$
\begin{aligned}
X_{1}\left(h_{1} / 2\right)= & \left(\begin{array}{r}
\left(\mathbf{m} \otimes \nu \cdots \mathbf{C}_{1} \cdot \nu \otimes \mathbf{m}\right)\left(\alpha_{1} \cosh \left(i r \alpha_{1} h_{1} / 2\right)+\beta_{1} \sinh \left(i r \alpha_{1} h_{1} / 2\right)\right)+ \\
\left(\mathbf{m} \otimes \nu \cdots \mathbf{C}_{1} \cdots \mathbf{n} \otimes \mathbf{m}\right) \sinh \left(i r \alpha_{1} h_{1} / 2\right)
\end{array}\right) \\
& \times i r e^{i r \beta_{1} h_{1} / 2} \\
Y_{1}\left(h_{1} / 2\right)= & \left(\begin{array}{c}
\left(\mathbf{m} \otimes \nu \cdots \mathbf{C}_{1} \cdots \nu \otimes \mathbf{m}\right)\left(\alpha_{1} \sinh \left(i r \alpha_{1} h_{1} / 2\right)+\beta_{1} \cosh \left(i r \alpha_{1} h_{1} / 2\right)\right)+ \\
\left(\mathbf{m} \otimes \nu \cdots \mathbf{C}_{1} \cdot \mathbf{n} \otimes \mathbf{m}\right) \cosh \left(i r \alpha_{1} h_{1} / 2\right)
\end{array}\right) \\
& \times i r e^{i r \beta_{1} h_{1} / 2} .
\end{aligned}
$$

b) Multiple roots

$$
\begin{aligned}
X_{1}\left(h_{1} / 2\right)= & \left(\gamma_{1}\left(\mathbf{m} \otimes \nu \cdots \mathbf{C}_{1} \cdot \nu \otimes \mathbf{m}\right)+\left(\mathbf{m} \otimes \nu \cdots \mathbf{C}_{1} \cdot \mathbf{n} \otimes \mathbf{m}\right)\right) i r e^{i r \gamma_{1} h_{1} / 2} \\
Y_{1}\left(h_{1} / 2\right)= & \left(\left(1+i r \gamma_{1} h_{1} / 2\right)\left(\mathbf{m} \otimes \nu \cdots \mathbf{C}_{1} \cdot \nu \otimes \mathbf{m}\right)+i r h_{1} / 2\left(\mathbf{m} \otimes \nu \cdots \mathbf{C}_{1} \cdots \mathbf{n} \otimes \mathbf{m}\right)\right) \\
& \times i r e^{i r \gamma_{1} h_{1} / 2}
\end{aligned}
$$

Now, Eq. (5.1) allows us to express (up to a multiplier) coefficients $C_{1}$ and $C_{2}$ in terms of the following equation:

$$
\vec{T}_{1}\left(h_{1} / 2\right) \times \vec{C}=0
$$

where

$$
\vec{T}_{1}\left(h_{1} / 2\right)=\left(-Y_{1}\left(h_{1} / 2\right) ; X_{1}\left(h_{1} / 2\right)\right) .
$$

It is clear from (5.8) that two-dimensional vector $\vec{T}_{1}\left(h_{1} / 2\right)$ is collinear with vector $\vec{C}$.

5.3. Boundary condition at the interface between the $n$-th layer and the substrate. Contact boundary conditions at this interface can be expressed in the form:

$$
\vec{V}_{n}\left(-h_{n} / 2\right) \cdot \vec{W}_{n+1}(0)=0
$$

where

$$
\vec{V}_{n}\left(-h_{n} / 2\right)=\left(u_{n}\left(-h_{n} / 2\right), t_{n}\left(-h_{n} / 2\right)\right)
$$

and

$$
\vec{W}_{n+1}(0)=\left(-t_{n+1}(0), u_{n+1}(0)\right) .
$$

In (5.12), $t_{n+1}(0)=\left|\mathbf{t}_{n+1}(0) e^{-i r(\mathbf{n} \cdot \mathbf{x}-c t)}\right|$ and $\mathbf{t}_{n+1}(0)$ is defined by (3.6). Taking into account (5.11), (5.12), condition (5.10) expresses collinearity of the vectors $\vec{V}_{n}$ and $\left(u_{n+1}(0), t_{n+1}(0)\right)$, the latter is equivalent to continuity of the displacements and surface tractions across the interface. In (5.12) it is assumed that in the local coordinate system for the substrate, the interface plane is represented by the equation $\nu \cdot \mathbf{x}=0$.

5.4. Secular equation for Love waves. Taking into account Eqs. (5.4), (5.8), (5.10), and (5.12), the secular equation for the MTM-method can be written in the form:

$$
\vec{W}_{n+1}(0) \cdot\left(\left(\prod_{k=2}^{n} \mathbf{M}_{k}\left(-h_{k} / 2\right) \cdot \mathbf{M}_{k}^{-1}\left(h_{k} / 2\right)\right) \cdot \mathbf{M}_{1}\left(-h_{1} / 2\right)\right) \cdot \vec{T}_{1}\left(h_{1} / 2\right)=0
$$

Equation (5.13) is the secular equation that we are seeking for Love waves. 
5.5. Secular equation for horizontally polarized shear waves in laminated plates. In this section we derive secular equations for horizontally polarized shear waves propagating in a laminated plate containing $n$ layers $(n>1)$. Outer surfaces of the plate are assumed to be traction-frec, clamped, or mixed (one outer surface is traction-free and the other is clamped).

a) Laminated plate with free outer surfaces. For such a plate, boundary conditions at the outer surfaces are as follows:

$$
\left\{\begin{array}{l}
\mathbf{t}_{1}\left(h_{1} / 2\right) \equiv \nu \cdot \mathbf{C}_{1} \cdot \nabla \mathbf{u}=0 \\
\mathbf{t}_{n}\left(-h_{n} / 2\right) \equiv \nu \cdot \mathbf{C}_{n} \cdot \nabla \mathbf{u}=0
\end{array}\right.
$$

Applying the MTM method and exploiting arguments similar to those used in the previous section yields the secular equation in the form:

$$
\vec{T}_{n}\left(-h_{n} / 2\right) \cdot \mathbf{M}_{n}^{-1}\left(h_{n} / 2\right) \cdot\left(\prod_{k=2}^{n-1} \mathbf{M}_{k}\left(-h_{n} / 2\right) \cdot \mathbf{M}_{k}^{-1}\left(h_{k} / 2\right)\right) \cdot \mathbf{M}_{1}\left(-h_{1} / 2\right) \cdot \vec{T}_{1}\left(h_{1} / 2\right)=0,
$$

where two-dimensional vectors $\vec{T}_{1}$ and $\vec{T}_{n}$ corresponding to boundary conditions (5.14) are:

$$
\begin{aligned}
\vec{T}_{1}\left(h_{1} / 2\right) & =\left(-Y_{1}\left(h_{1} / 2\right) ; X_{1}\left(h_{1} / 2\right)\right) \\
\vec{T}_{n}\left(-h_{n} / 2\right) & =\left(X_{n}\left(-h_{n} / 2\right) ; Y_{n}\left(-h_{n} / 2\right)\right) .
\end{aligned}
$$

Components $X_{k}, Y_{k}, k=1, n$ in (5.16) are defined by (5.6), (5.7).

b) Laminated plate with clamped outer surfaces. For such a plate, boundary conditions on the outer surfaces become:

$$
\left\{\begin{array}{l}
\mathbf{u}_{1}\left(h_{1} / 2\right)=0 \\
\mathbf{u}_{n}\left(-h_{n} / 2\right)=0
\end{array}\right.
$$

As before, applying the MTM method yields the secular equation in the form:

$$
\overrightarrow{D_{n}}\left(-h_{n} / 2\right) \cdot \mathbf{M}_{n}^{-1}\left(h_{n} / 2\right) \cdot\left(\prod_{k=2}^{n-1} \mathbf{M}_{k}\left(-h_{n} / 2\right) \cdot \mathbf{M}_{k}^{-1}\left(h_{k} / 2\right)\right) \cdot \mathbf{M}_{1}\left(-h_{1} / 2\right) \cdot \overrightarrow{D_{1}}\left(h_{1} / 2\right)=0
$$

where vectors $\overrightarrow{D_{1}}\left(h_{1} / 2\right), \overrightarrow{D_{n}}\left(-h_{n} / 2\right)$ have the form:

$$
\begin{aligned}
\overrightarrow{D_{1}}\left(h_{1} / 2\right) & =\left(-U_{1}\left(h_{1} / 2\right) ; S_{1}\left(h_{1} / 2\right)\right) \\
\overrightarrow{D_{n}}\left(-h_{n} / 2\right) & =\left(S_{n}\left(-h_{1} / 2\right) ; U_{n}\left(-h_{n} / 2\right)\right) .
\end{aligned}
$$

In (5.19) components $S_{k} ; U_{k}, k=1, n$ for aliquant roots according to (4.1) are:

$$
\begin{aligned}
& S_{k}\left( \pm h_{k} / 2\right)= \pm \sinh \left(i r \alpha_{k} h_{k} / 2\right) \\
& U_{k}\left( \pm h_{k} / 2\right)=\cosh \left(i r \alpha_{k} h_{k} / 2\right)
\end{aligned}
$$

with $\alpha_{k}$ defined by (4.2).

Components $S_{k} ; U_{k}, k=1, n$ for multiple roots according to (4.7) have the form:

$$
\begin{gathered}
S_{k}\left( \pm h_{k} / 2\right)=1 \\
U_{k}\left( \pm h_{k} / 2\right)= \pm i r h_{k} / 2 .
\end{gathered}
$$


c) Laminated plate with one clamped and one traction-free outer surface. For such a plate, boundary conditions are:

$$
\left\{\begin{array}{l}
\mathbf{t}_{1}\left(h_{1} / 2\right)=0 \\
\mathbf{u}_{n}\left(-h_{n} / 2\right)=0
\end{array}\right.
$$

where it is assumed that the upper surface of the plate is traction-free and the bottom is clamped. For this case the secular equation is:

$\overrightarrow{D_{n}}\left(-h_{n} / 2\right) \cdot \mathbf{M}_{n}^{-1}\left(h_{n} / 2\right) \cdot\left(\prod_{k=2}^{n-1} \mathbf{M}_{k}\left(-h_{n} / 2\right) \cdot \mathbf{M}_{k}^{-1}\left(h_{k} / 2\right)\right) \cdot \mathbf{M}_{1}\left(-h_{1} / 2\right) \cdot \vec{T}_{1}\left(h_{1} / 2\right)=0$.

Modification of Eq. (5.23) for the case when the upper surface is clamped and the bottom is traction-free is obvious.

REMARK 5.1. The left-hand sides of secular equations (5.13), (5.15), (5.18), and (5.23) can be regarded as implicit equations with respect to wave number $r$ at the fixed phase frequency $\omega$, and vice versa. Using in these equations the following relation:

$$
r=\frac{\omega^{\prime}}{c}
$$

where $\omega$ is the phase frequency, we arrive at equations yielding the dispersion relations in terms of phase frequency and phase speed.

\section{Some analytical solutions.}

6.1. An orthotropic layer on orthotropic substrate. Let vectors $\nu, \mathbf{n}$, and $\mathbf{m}$ coincide with the principle elasticities of both the layer and the substrate. For these orientations the Christoffel parameters $\gamma_{k}$ become

$$
\gamma_{k}=(-1)^{k+1} i \sqrt{\frac{\mathbf{m} \otimes \mathbf{n} \cdot \mathbf{C}_{k} \cdot \mathbf{n} \otimes \mathbf{m}-\rho_{k} c^{2}}{\mathbf{m} \otimes \nu \cdot \mathbf{C}_{k} \cdot \nu \otimes \mathbf{m}}}, \quad k=1,2 .
$$

In (6.1) and further in this subsection, index 1 is referred to as the layer, and 2 is referred to as the substrate.

REMARK 6.1. a) Expression (6.1) 1 shows that multiple roots for the layer can arise only at the phase speed coinciding with the speed of the transverse bulk wave propagating in the direction defined by the vector $\mathbf{n}$ and polarized in the $\mathbf{m}$ direction.

b) Although some analytical results for one orthotropic layer lying on an orthotropic substrate were obtained in [2], no explicit dispersion equation has been derived yet.

Scalar amplitude of the surface tractions $t_{1}\left(x^{\prime}\right) \equiv\left|\mathbf{t}_{1}\left(x^{\prime}\right) e^{-i r(\mathbf{n} \cdot \mathbf{x}-c t)}\right|$ acting on a plane $\nu \cdot \mathbf{x}=x^{\prime}$ in the layer is as follows:

a) Aliquant roots

$$
t_{1}\left(x^{\prime}\right)=i r \gamma_{1}\left(\mathbf{m} \otimes \nu \cdot \mathbf{C}_{1} \cdot \nu \otimes \mathbf{m}\right)\left(C_{1} \cosh \left(i r \gamma_{1} x^{\prime}\right)+C_{2} \sinh \left(i r \gamma_{1} x^{\prime}\right)\right) .
$$

b) Multiple roots $\left(\gamma_{1}=0\right)$

$$
t_{1}=i r\left(\mathbf{m} \otimes \nu \cdots \mathbf{C}_{1} \cdot \nu \otimes \mathbf{m}\right) C_{2} .
$$


Scalar amplitude of the surface tractions $t_{2}(0) \equiv\left|\mathbf{t}_{2}(0) e^{-i r(\mathbf{n} \cdot \mathbf{x}-c t)}\right|$ acting on the interface plane $\nu \cdot \mathbf{x}=0$ in the substrate is as follows:

$$
\left.t_{2}(0)\right|_{\nu \cdot \mathbf{x}=0}=i r \gamma_{2} x\left(\mathbf{m} \otimes \nu \cdots \mathbf{C}_{2} \cdot \nu \otimes \mathbf{m}\right) C_{3} .
$$

Proposition 6.1. No Love wave can propagate in a system composed of a single orthotropic layer lying on an orthotropic substrate when multiple roots in the Christoffel equation for the layer arise.

Proof. Expressions (4.5), (6.1) show that multiple roots $\gamma_{1}$ for the layer can only be zeros. For such a case the traction-free boundary conditions (5.7), (5.8) along with expression (6.3) yield

$$
C_{2}=0 \text {. }
$$

Interface boundary condition (5.10) along with (6.3)-(6.5) result in

$$
C_{3}=0 \text {. }
$$

But the latter condition implies that there are no displacements at the interface, and this leads to:

$$
C_{1}=0 \text {. }
$$

Conditions (6.5)-(6.7) complete the proof, since at all zeros $C_{k}, k=1,2,3$, no surface wave propagates.

Excluding multiple roots. we can confine ourselves to the aliquant roots. Applying (5.2) to the orthotropic layer yields

$$
\mathbf{M}_{1}\left(x^{\prime}\right)=\left(\begin{array}{c|c}
\sinh \left(i r \gamma_{1} x^{\prime}\right) & \cosh \left(i r \gamma_{1} x^{\prime}\right) \\
\hline i r \gamma_{1}\left(\mathbf{m} \otimes \nu \cdot \mathbf{C}_{1} \cdot \nu \otimes \mathbf{m}\right) \cosh \left(i r \gamma_{1} x^{\prime}\right) & i r \gamma_{1}\left(\mathbf{m} \otimes \nu \cdot \mathbf{C}_{1} \cdot \nu \otimes \mathbf{m}\right) \sinh \left(i r \gamma_{1} x^{\prime}\right)
\end{array}\right) .
$$

Up to a scalar multiplier $\operatorname{ir} \gamma_{1}\left(\mathbf{m} \otimes \nu \cdots \mathbf{C}_{1} \cdot \nu \otimes \mathbf{m}\right)$, vector $\vec{T}_{1}$ defined by (5.9) admits representation:

$$
\vec{T}_{1}\left(h_{1} / 2\right)=\left(-\sinh \left(i \gamma_{1} h_{1} / 2\right) ; \cosh \left(i r \gamma_{1} h_{1} / 2\right)\right) .
$$

Similarly, vector $\vec{W}_{2}$ defined by $(5.12)$ is:

$$
\vec{W}_{2}=\left(-i r \gamma_{2} \mathbf{m} \otimes \nu \cdot \mathbf{C}_{2} \cdot \nu \otimes \mathbf{m} ; 1\right) .
$$

Substituting (6.8)-(6.10) into Eq. (5.13) after some transformations yields

$$
\omega=\frac{c}{\gamma_{1} h_{1}}\left(\arctan \left(i \frac{\zeta_{2}}{\zeta_{1}}\right)+n \pi\right), \quad n=0,1,2, \ldots,
$$

where $\zeta_{k}=\gamma_{k}\left(\mathbf{m} \otimes \nu \cdots \mathbf{C}_{k} \cdot \nu \otimes \mathbf{m}\right), \quad k=1.2$.

Proposition 6.2. a) In the regarded system a Love wave can propagate if and only if the phase speed belongs to the interval $c \in\left(c_{1}^{T} ; c_{2}^{T}\right)$ (for this speed interval all the roots of the Christoffel equation for the layer are aliquant).

b) At the fixed frequency $\omega$ there is a finite number of Love waves propagating with different phase speeds $c \in\left(c_{1}^{T} ; c_{2}^{T}\right)$, where

$$
c_{k}^{T}=\sqrt{\frac{\mathbf{m} \otimes \mathbf{n} \cdot \mathbf{C}_{k} \cdot \mathbf{n} \otimes \mathbf{m}}{\rho_{k}}}, \quad k=1,2
$$

are the speeds of the corresponding shear bulk waves. 
c) At the fixed phase speed $c \in\left(c_{1}^{T} ; c_{2}^{T}\right)$ there is a denumerable number of Love waves propagating with different frequencies $\omega$.

Proof. To prove a), assume that $c_{1}^{T}<c_{2}^{T}$; then the corresponding speed interval is not empty. Analysis of expression (6.11) shows that at the phase speed belonging to the interval $\left(c_{1}^{T} ; c_{2}^{T}\right)$, the Christoffel parameter $\gamma_{1}$ defined by $(6.1)_{1}$ is negative real, while $\gamma_{2}$ defined by $(6.2)_{2}$ is purely imaginary with negative imaginary part. Substituting these $\gamma_{k}, k=1,2$ into (6.11) results in positive values for the phase frequency $\omega$. Supposing now that $c<c_{1}^{T}$ leads to

$$
\omega=-\frac{c}{\left|\gamma_{1}\right| h_{1}} \tanh ^{-1}\left(\left|\frac{\zeta_{2}}{\zeta_{1}}\right|\right) .
$$

It is clear that $\left(6.11^{\prime}\right)$ gives negative values for $\omega$, which is impossible. Thus, statement a) is proved. Other statements flow out directly from analysis of (6.11).

Corollary. No Love waves can propagate if $c_{1}^{T}>c_{2}^{T}$.

6.2. Two orthotropic layers on orthotropic substrate (aliquant roots). Adopting the developed notation, we arrive at the following expression for multiplication of the transfer matrices:

$$
\mathbf{M}_{2}\left(-h_{2} / 2\right) \cdot \mathbf{M}_{2}^{-1}\left(h_{2} / 2\right) \cdot \mathbf{M}_{1}\left(-h_{1} / 2\right)=\left(\begin{array}{ll}
a_{11} & a_{12} \\
a_{21} & a_{22}
\end{array}\right)
$$

where

$$
\begin{aligned}
a_{11} & =-i\left(\cos \left(\xi_{2}\right) \sin \left(\frac{1}{2} \xi_{1}\right)+\frac{\zeta_{1}}{\zeta_{2}} \sin \left(\xi_{2}\right) \cos \left(\frac{1}{2} \xi_{1}\right)\right), \\
a_{12} & =\cos \left(\xi_{2}\right) \cos \left(\frac{1}{2} \xi_{1}\right)-\frac{\zeta_{1}}{\zeta_{2}} \sin \left(\xi_{2}\right) \sin \left(\frac{1}{2} \xi_{1}\right), \\
a_{21} & =-i r \zeta_{2}\left(\sin \left(\xi_{2}\right) \sin \left(\frac{1}{2} \xi_{1}\right)-\frac{\zeta_{1}}{\zeta_{2}} \cos \left(\xi_{2}\right) \cos \left(\frac{1}{2} \xi_{1}\right)\right), \\
a_{22} & =r \zeta_{2}\left(\sin \left(\xi_{2}\right) \cos \left(\frac{1}{2} \xi_{1}\right)+\frac{\zeta_{1}}{\zeta_{2}} \cos \left(\xi_{2}\right) \sin \left(\frac{1}{2} \xi_{1}\right)\right), \\
\xi_{k} & =r \gamma_{k} h_{k}, \quad \zeta_{k}=\gamma_{k}\left(\mathbf{m} \otimes \nu \cdot \mathbf{C}_{k} \cdot \nu \otimes \mathbf{m}\right), \quad k=1,2,3 .
\end{aligned}
$$

In (6.14) indices 1 and 2 are referred to as layers and index 3 is referred to as the substrate.

Expressions (6.9) and (6.10) for vectors $\vec{T}_{1}$ and $\vec{W}_{3}$ remain the same, but with the obvious change of indices. Substituting (6.13), (6.14) into Eq. (5.13) yields the secular equation in the form:

$$
\sin \xi_{2}\left(\cos \xi_{1}+i \frac{\zeta_{3} \zeta_{1}}{\zeta_{2}^{2}} \sin \xi_{1}\right)-\cos \xi_{2}\left(i \frac{\zeta_{3}}{\zeta_{2}} \cos \xi_{1}-\frac{\zeta_{1}}{\zeta_{2}} \sin \xi_{1}\right)=0 .
$$

In contrast to the previous case, there is no general explicit solution for Eq. (6.15) which would resemble (6.11).

6.3. Two orthotropic substrates with an orthotropic layer between them. Taking into account Corollary 1 from Proposition 3.1, we must exclude considering multiple roots for both half-spaces. On the other hand, according to Remark 6.1a, multiple roots for the layer arise if and only if the phase speed coincides with the speed of the transverse bulk 
wave and the corresponding Christoffel parameter $\gamma_{2}$ vanishes. Now, applying arguments analogous to those used in proof of Proposition 6.1, we arrive at

PROPOSITION 6.3. No Love wave can propagate in a system composed of two orthotropic half-spaces and an orthotropic layer between them when multiple roots in the Christoffel equation for the layer arise.

Excluding multiple roots, in the remaining part of this section we will consider the case of aliquant roots. Assuming that in both substrates surface waves attenuate with depth, we are forced to regard the phase speed belonging to the interval

$$
c<\min \left(c_{1}^{T} ; c_{3}^{T}\right)
$$

where, as before, $c_{1}^{T}, c_{3}^{T}$ stand for velocities of bulk horizontally polarized shear waves propagating in a direction defined by the vector $\mathbf{n}$. Condition (6.16) ensures the Christoffel parameters $\gamma_{1}, \gamma_{3}$ to be imaginary.

Remark 6.2. Attenuation with depth in the "upper" half-space (when $x^{\prime} \rightarrow+\infty$ ) is achieved by choosing $\gamma_{1}$ with positive imaginary part.

Taking the limit $h_{1} \rightarrow \infty$ in Eq. (6.15) and keeping in mind Remark 6.2 yields the desired dispersion relation:

$$
\omega=\frac{c}{\gamma_{2} h_{2}}\left(\arctan \left(i \frac{\zeta_{2}\left(\zeta_{3}-\zeta_{1}\right)}{\zeta_{2}^{2}-\zeta_{1} \zeta_{3}}\right)+n \pi\right), \quad n=m, m+1, m+2, \ldots
$$

Parameter $m$ appearing in the right-hand side of (6.17) is chosen for obtaining positive values for the frequency $\omega$. Later in this subsection this parameter will be specified explicitly. It is obvious that at $\zeta_{1}=0$ (vacuum), dispersion relation (6.17) transforms into relation $(6.11)$.

PROPOSITION 6.4. a) In the regarded system, a Love wave can propagate if and only if the phase speed belongs to the interval

$$
c \in\left(c_{2}^{T} ; \min \left(c_{1}^{T}, c_{3}^{T}\right)\right) .
$$

b) At the fixed frequency $\omega$ there is a finite number of Love waves propagating with different phase speeds belonging to interval (6.18).

c) At the fixed phase speed from the interval (6.18), there is a denumerable number of Love waves propagating with different frequencies $\omega$.

Proof. a) If $c>\min \left(c_{1}^{T}, c_{3}^{T}\right)$, then no Love wave can propagate, since attenuation in the contacting substrates cannot be achieved. Suppose now that $c<\min \left(c_{1}^{T} ; c_{2}^{T} ; c_{3}^{T}\right)$; then all the Christoffel parameters $\gamma_{k}$ become imaginary:

$$
\gamma_{1}=+i\left|\gamma_{1}\right| ; \quad \gamma_{2}= \pm i\left|\gamma_{2}\right| ; \quad \gamma_{3}=-i\left|\gamma_{3}\right| .
$$

In (6.19) Remark 6.2 is taken into consideration. Substituting (6.19) into (6.17) yields:

$$
\omega=-\frac{c}{\left|\gamma_{2}\right| h_{2}} \tanh ^{-1}\left(\frac{\left|\zeta_{2}\right|\left(\left|\zeta_{3}\right|+\left|\zeta_{1}\right|\right)}{\left|\zeta_{2}\right|^{2}+\left|\zeta_{1}\right|\left|\zeta_{3}\right|}\right) .
$$

Thus, the right-hand side of (6.20) is negative, while the phase frequency $\omega$ should be positive. 
Now it remains to show that at the phase speed satisfying (6.18), the corresponding $\omega$ is positive. For this speed interval

$$
\gamma_{1}=+i\left|\gamma_{1}\right| ; \quad \gamma_{2}= \pm\left|\gamma_{2}\right| ; \quad \gamma_{3}=-i\left|\gamma_{3}\right|
$$

$\left(\gamma_{2}\right.$ is real). Substituting (6.21) into (6.17) yields

$$
\omega=\frac{c}{\left|\gamma_{2}\right| h_{2}}\left(\arctan \left(\frac{\left|\zeta_{2}\right|\left(\left|\zeta_{3}\right|+\left|\zeta_{1}\right|\right)}{\left|\zeta_{2}\right|^{2}-\left|\zeta_{1}\right|\left|\zeta_{3}\right|}\right)+n \pi\right), \quad n=m, m+1, m+2, \ldots,
$$

where the integer parameter $m$ appearing in the right-hand side of $\left(6.17^{\prime}\right)$ satisfies the following equation:

$$
m=-E n t\left(\pi^{-1} \arctan \left(\frac{\left|\zeta_{2}\right|\left(\left|\zeta_{3}\right|+\left|\zeta_{1}\right|\right)}{\left|\zeta_{2}\right|^{2}-\left|\zeta_{1}\right|\left|\zeta_{3}\right|}\right)\right) .
$$

Equation (6.22) ensures the phase frequency $\omega$ to be positive. In (6.22) Ent(...) stands for the integer part.

Proofs of assertions b) and c) are obvious.

Corollary. No Love waves can propagate if $c_{2}^{T}>\min \left(c_{1}^{T}, c_{3}^{T}\right)$.

REMARK 6.3. Results obtained in this subsection are important for practical applications, since they lead to a better understanding of the phenomenon of developing high frequency waveguides for Love waves propagating in a layer contacting with two substrates. These results also generalize conditions for the existence of surface waves resembling Stoneley waves, but with the horizontal shear polarization (Stoneley waves propagate on the interface between contacting half-spaces and attenuate with depth in both half-spaces).

7. Numerical solution. In this section we apply the developed MTM method for obtaining dispersion relations for Love waves propagating in a medium containing 10 alternating layers lying on a single crystal silicone ( $\mathrm{Si}$ ) substrate with orientation [100]; this crystal belongs to the orthorhombic (cubic) class of elastic symmetry. Mechanical properties of silicon needed for computations are as follows:

$$
C^{1212}=79.913 G P a ; \quad \rho=2339.9 \mathrm{~kg} / \mathrm{m}^{3} .
$$

Alternating layer coatings are silicon carbide ( $\mathrm{SiC})$ :

$$
C^{1212}=122.80 G P a ; \quad \rho=3100 \mathrm{~kg} / \mathrm{m}^{3},
$$

and silicon nitride $\left(\mathrm{Si}_{3} \mathrm{~N}_{4}\right)$ :

$$
C^{1212}=61.447 G P a ; \quad \rho=3290 \mathrm{~kg} / \mathrm{m}^{3} .
$$

These coatings (both isotropic) are quite often used in microelectronics. All the parameters in (7.1)-(7.3) are evaluated at room temperature.

In our computational model, each layer had 10 nanometers thickness, which is typical in some advanced microelectronic devices. Such a small thickness, however, is enough for using equations of continuum mechanics, since about 100 atoms can be placed along the depth of a layer. 


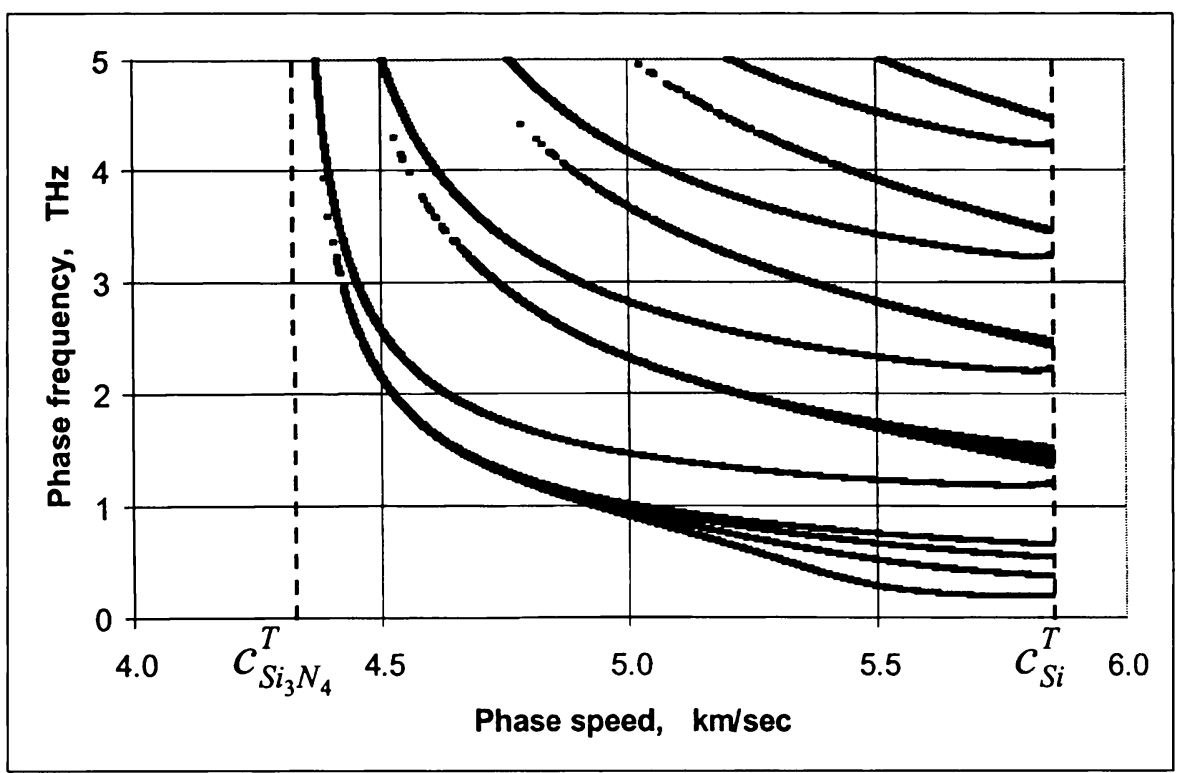

FIG. 1. Dispersion curves

Numerical implementation of the MTM method used in this section is based on a search of the zeros of Eq. (5.13) at a given value of the phase speed $c \in\left(0 ; c_{n+1}^{T}\right)$. The first $N$ zeros, representing phase frequency w (see Remark 5.1), were searched in the interval $(0 ; \infty)$ with the successive refinement. Computer code was based on the high precision numerical package, allowing us to retain up to 512 decimal digits in mantissas.

The first several dispersion curves for the regarded medium are plotted in Fig. 1. Vertical dashed lines in Fig. 1 refer to velocities of the corresponding transverse bulk waves.

Then, to check sensitivity of the developed method to identify properties of layers and the substrate, we increased by $10 \%$ the thickness of a particular layer, leaving the other layers unaltered, and applying the MTM method, calculated the difference

$$
\Delta \omega_{k}(c)=\omega_{k}(c)-\omega(c)
$$

between the lower branch of the dispersion curve $\omega_{k}(c)$ for a medium with the disturbed $k$-th layer, and the lower branch $\omega(c)$ for the undisturbed medium. These data are plotted in Fig. 2.a. Analogous results are obtained at the simultaneous increase by $10 \%$ of the density and shear modulus of a particular layer and the substrate. These data are presented in Fig. 2.b.

The obtained data reveal that even for the most distant lavers and the substrate there is a possibility of determining variation of their properties due to a quite noticeable change of the lower branches of the dispersion curves.

Acknowledgements. The author thanks Professor I. Djeran-Maigre for valuable support, Dr. D. H. Bailey for the Portable High Performance Multiprecision Package, 

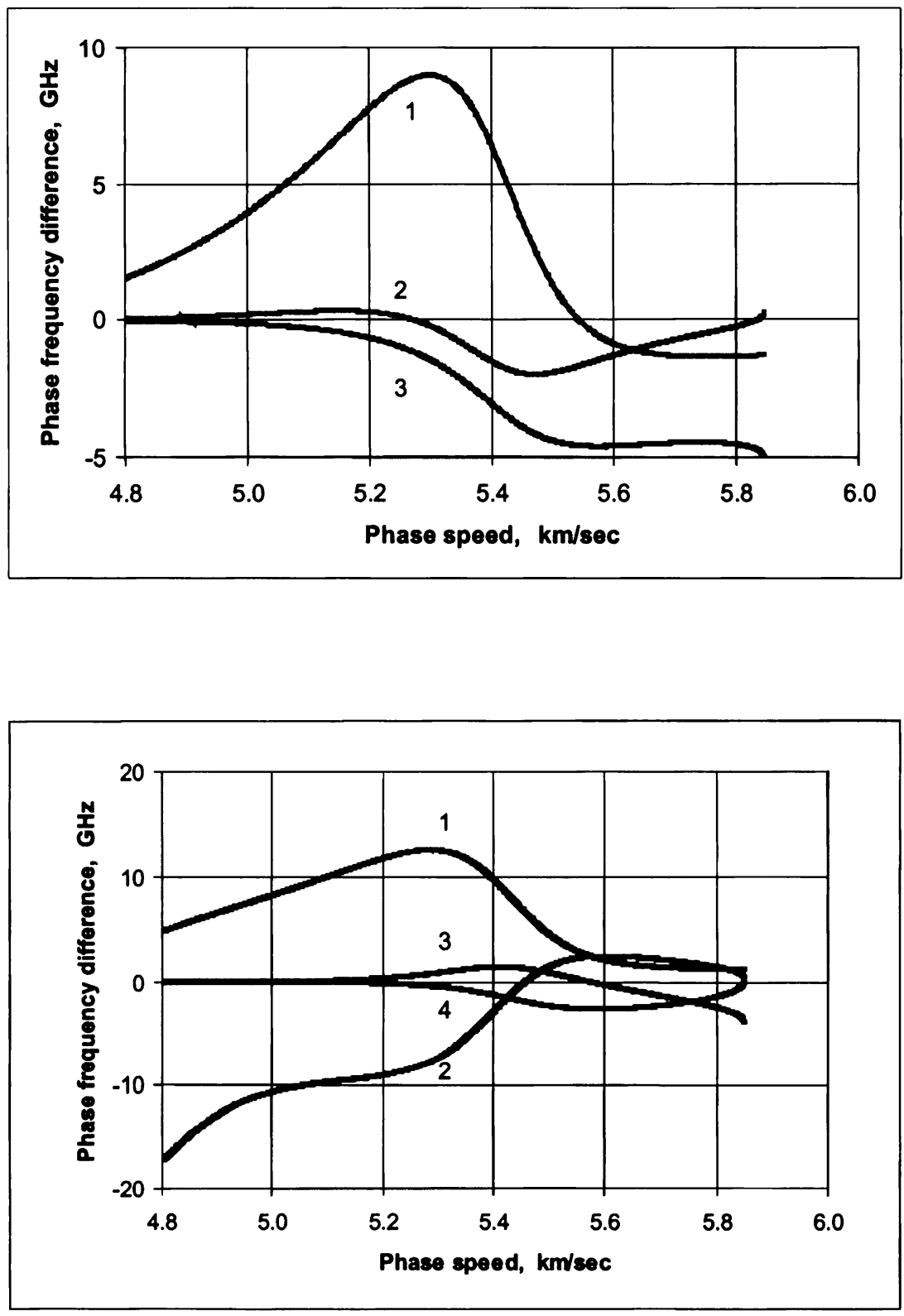

FIG. 2. Variation of the lower branch of the dispersion curve due to: a) $10 \%$ thickness increase; b) simultaneous $10 \%$ increase of density and shear modulus;

(1) $-7^{\text {th }}$ layer; (2) $-9^{\text {th }}$ layer; (3) $-10^{\text {th }}$ layer; (4) -substrate. 
RFFI Grant 04-01-00781, and Russian Academy of Sciences Program 13 for financial support.

\section{REFERENCES}

[1] A. E. H. Love, Some Problems of Geodynamics, Cambridge University Press, London, 1911

[2] E. Dieulesaint and D. Royer, Elastic Waves in Solids, Wiley, N. Y., 1980

[3] P. R. Sengupta and S. Nath, Surface waves in fiber-reinforced anisotropic elastic media, Sadhana 26, 363-370 (2001)

[4] W. T. Thomas, Transmission of elastic waves through a stratified solid medium, J. Appl. Phys. 21, 89-93 (1950)

[5] N. A. Haskell, Dispersion of surface waves on multilayered media, Bull. Seism. Soc. Am. 43, 17-34 (1953)

[6] L. Knopoff, A matrix method for elastic wave problems, Bull. Seism. Soc. Am. 54, 431-438 (1964)

[7] A. K. Mal and L. Knopoff, A differential equation for surface waves in layers with varying thickness, J. Math. Anal. Appl. 21, 431-441 (1968)

[8] J. W. Dunkin, Computation of model solutions in layered elastic media at high frequencies, Bull. Seism. Soc. Am. 55, 335-358 (1965)

[9] T. Kundu and A. K. Mal, Elastic waves in a multilayered solid due to a dislocation source, Wave Motion 7, 459-471 (1985)

[10] R. B. Evans, The decoupling of seismic waves, Wave Motion 8, 321-328 (1986)

[11] M. Castaings and B. Hosten, Delta operator technique to improve the Thomson-Haskell method stability for propagation in multilayered anisotropic absorbing plates, J. Acoust. Soc. Am. (1994)

[12] M. J. S. Lowe, Matrix techniques for modeling ultrasonic waves in multilayered media. IEEE Trans. Ultrasonics, Ferroelectrics, and Frequency Control 42, 525-542 (1995)

[13] M. Mallah, L. Philippe, and A. Khater, Numerical computations of elastic wave propagation in anisotropic thin films deposited on substrates, Comp. Mater. Sci. 15, 411-421 (1999)

[14] R. Wobst, The generalized eigenvalue problem and acoustic surface wave computations, Computing 39, 57-69 (1987)

[15] M. E. Gurtin, The Linear Theory of Elasticity, In: Handbuch der Physik, Bd. VIa/2, Springer, Berlin, 1972

[16] S. V. Kuznetsov, "Forbidden" planes for Rayleigh waves, Quart. Appl. Math, 60, 87-97 (2002)

[17] S. V. Kuznetsov, Subsonic Lamb waves in anisotropic plates, Quart. Appl. Math. 60, 577--587 (2002)

[18] S. V. Kuznetsov, Surface waves of Non-Rayleigh type, Quart. Appl. Math. 61, 575-582 (2003) 